\title{
Das Thema Diabetes \\ aus verschiedenen Blickwinkeln
}

Wie in der vorletzten Ausgabe angekündigt, haben wir ein Schwerpunktheft zum Thema „Diabetes und Reisen“ zusammengestellt. Wir glauben, dass die Mitarbeit verschiedener Fachgesellschaften in „unserer“ Zeitschrift Flugmedizin Tropenmedizin Reisemedizin die einzigartige Gelegenheit bietet, ein Thema aus verschiedenen Blickwinkeln anzugehen - zum Vorteil für alle.

Das Thema „Diabetes“ hat sich hierfür natürlich angeboten: Die Erkrankung ist weit verbreitet, dementsprechend häufig sind auch Reisende betroffen. Andererseits gibt es auch Besonderheiten, etwa bei Patienten in den Tropen bzw. aus den Tropen. Die Urlaubsplanung eines Diabetikers erfordert Vorbereitung und Umsicht, viele Aspekte sind dabei zu beachten: Angefangen von den Empfehlungen zur Prophylaxe vor Reiseantritt, der Einstellung des Diabetes unterwegs, Erkrankungen nach der Rückkehr und vielem anderen mehr - nicht alles konnten wir in unserem Heft berücksichtigen.

Wir haben daher folgende Auswahl getroffen: Dr. Burkhard Rieke berichtet über Impfungen und Prophylaxeempfehlungen beim Diabetiker - Fragen, die jedem reisemedizinisch tätigen Kollegen gestellt werden können. Dr. Bodo Gutt, Dr. Rainald Fischer und Kollegen berichten über ein Spezialthema: Worauf ist beim Diabetiker bei Höhenaufenthalten $\mathrm{zu}$ achten? Dieses ist - soweit ich das beurteilen kann - eine ganz hervorragende Zusammenfassung, wie man sie in dieser Form nicht so einfach findet. Dr. Andreas Adrian und Dr. Jörg Siedenburg haben als Flugmediziner die klinischen und flugmedizinischen Aspekte beim Diabetiker beleuchtet - ebenfalls relevant für jeden Reisemediziner. Schließlich ist auch auf Besonderheiten bei Migranten hinzuweisen, so haben Südasia- ten eine bedeutend höhere Prävalenz für ein metabolisches Syndrom, Diabetes, Insulinresistenz mit Hyperinsulinämie und Dyslipidämien, sie weisen niedrigere HDL-Spiegel, höhere Lipoprotein-a-Werte, höhere Triglyzeride und eine erhöhte Thromboseneigung mit erhöhten Plasminogen-Aktivator-Inhibitor-1-Spiegeln auf. Daneben gibt es auch Besonderheiten eines Diabetes in den Tropen, die Dr. Stefan Schmiedel in seinem Beitrag erläutert. Und last, not least möchte ich Sie auch auf unsere erste Kasuistik hinweisen. Hier ist vielleicht noch anzufügen, dass neuerdings diskutiert wird, ob HHV-8Infektionen ursächlich an der Entstehung eines Diabetes in Afrika beteiligt sein könnten (siehe z.B. Sobngwi E, Choukem SP, Agbalika F et al. Ketosis-prone type 2 diabetes mellitus and human herpesvirus 8 infection in sub-saharan africans. JAMA 2008; 299: 2770-2776).

Ich möchte mich bei den Autoren für ihre hochkompetenten Beiträge bedanken und hoffe, dass Sie dieses Heft mit Gewinn lesen.

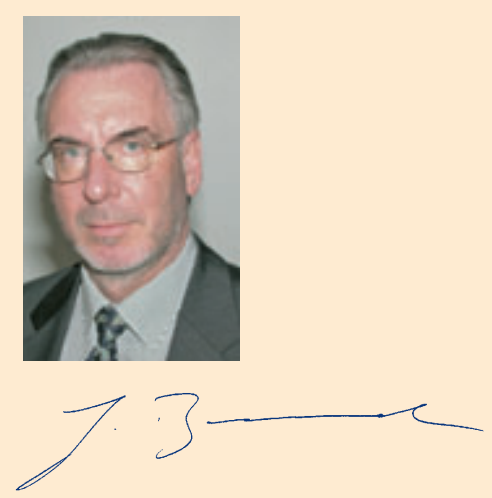

Prof. Dr. Gerd-Dieter Burchard, Hamburg 